\title{
Prototype Monitoring System for Power Line Inspection by Means of a PandaBoard
}

\author{
Rupert Gouws and Tjaart Visser \\ Faculty of Engineering, North-West University, Potchefstroom 2531, South Africa
}

Received: March 24, 2013 / Accepted: July 10, 2013 / Published: January 31, 2014.

\begin{abstract}
The national energy supplier (Eskom in South Africa) supplies electricity through thousands-of-kilometers of overhead power lines. The current methods of inspection of these overhead power lines are infrequent and expensive. In this paper, the authors present the development of a prototype monitoring system for power line inspection in South Africa. The developed prototype monitoring system collects data (information) from the overhead power lines, is remotely accessible and fits into a power line robot. The prototype monitoring system makes use of a PandaBoard ${ }^{\circledR}$ (SBC) with GPS receiver and 5 MP camera to collect data. Hardware fatigue is the biggest problem faced on the overhead power lines and is captured by means of the 5 MP camera and is displayed on a website hosted by the PandaBoard $^{\circledR}$ via Wi-Fi. The monitoring system has low power consumption, is light weight, compact and easily collects data. The data obtained from the prototype monitoring system was satisfactory and provides an improved solution for monitoring power lines for Eskom in South Africa.
\end{abstract}

Key words: Inspection robot, monitoring system, PandaBoard, transmission line inspection.

\section{Introduction}

Eskom has thousands-of-kilometers of transmission lines running across South Africa [1]. All of these lines have to be patrolled and maintained. Eskom is currently doing foot patrols and fast helicopter inspections annually together with detail/slow helicopter inspections every two years on their lines.

These methods of overhead power line inspection has been implemented for a long time now and it is expensive and infrequent [2, 3]. When a key component on a tower starts failing, like a wire connector, it has the tendency to fail completely over a short period of time. Almost like the snow ball effect. Although multiple departments inspect the lines, some lines are routed in remote areas (across mountains, over rivers, etc.) which mean inspection is more difficult and more time consuming $[4,5]$.

Eskom mostly collects detailed photos [6] during

Corresponding author: Rupert Gouws, Ph.D., researcher, research fields: energy engineering, electrical machines and control.E-mail: rupert.gouws@nwu.ac.za. these inspections coupled with an occasional infrared photo (usually during slow helicopter inspection). By using a helicopter to collect these photos results in high costs to Eskom. Hijacking has also become a big issue during foot patrols. The use of an inspection robot for maintenance purposes, will contribute towards safety around live lines by not requiring a person to climb onto the live wire for close inspection [7].

This project is the first step towards an implementable inspection robot for Eskom. This attempted monitoring system is a baseline to further expand and improve towards the complete monitoring solution intended by this project. Some key features have been identified to aim for in this first attempt as indicated in the list shown below:

- The monitoring system must incorporate sensors to collect evidence from the line;

- The sensors must collect information at a high rate to ensure that data from each part of the line are collected to prevent blind spots which may overlook key components that can cause line failure; 
- The monitoring system will make use of a wireless communication device to transmit the newly collected information to a remote device;

- The wireless broadcasting range at this stage does not need to be very far as it is only essential to have wireless connectivity to form a base communication system. Range boosters can easily be inserted in the future to increase the range;

- The monitoring system must be integrated with the inspection robot and use its battery source without disrupting the robot's operation [8].

The aim of the project was to successfully complete an integrated hardware system which will allow the collection of detailed evidence (HD photos) together with sensors that provide location (time and GPS coordinates). The system also has to incorporate software which will utilize the hardware to a point where the intervals of data collection cover the complete line and then host the evidence, wirelessly, over a network $[6,8]$.

\section{Materials and Method}

\subsection{Technical Survey and Recommendations}

At Eskom in their central transmission division located in Simmerpan (South Africa), the following problems have been identified as problems which Eskom face on their overhead transmission lines:

- Visible hardware problems-These include all the physical fatigue noticeable by the human eye. Ground patrols use HD cameras to take pictures of each overhead power line tower and then take it to their office to process and look for suspicious components;

- Non visible hardware problems-The two invisible killers are weak connections and corona. Both are detectable by an infrared camera. Corona is also detectable with an ultraviolet camera. These two camera technologies are very expensive;

- Wire problems-Overhead power lines have to comply with clearance standards [3, 8]. Breaching these limits results in flashovers. To measure these distances, Eskom uses a lidar scanner to create a 3D model which shows all the distances. But just like the infrared and ultraviolet cameras, lidar systems are very expensive;

- High inspection costs-The current aerial inspection cost and foot patrol inspections of the Eskom transmission lines are too expensive.

After understanding what problems Eskom face, one can not help but wonder if the alternative solutions do not really provide the solution that Eskom needs. Eskom is currently spending a lot of money just to patrol the transmission lines. By buying a robot which specializes in temporary close inspection and small repairs (like the LineScout ${ }^{\circledR}[9,10]$ and Expliner), Eskom is only shifting towards better safety for humans and not towards saving money during foot patrols whilst improving safety. Thus, the LineScout ${ }^{\circledR}$ and Expliner do not meet the solution Eskom require.

The cable crawler, just like the LineScout ${ }^{\circledR}$ and Expliner, is a very big and heavy robot. Its monitoring system is not close to being finished, so it is too early to tell whether the developers are heading in the right direction. The high-wire acrobat is showing potential for future use by Eskom, but the high cost and the fact that field testing will only start in 2014, eliminates the high-wire acrobat as a current competitor.

If a monitoring system, which only looks for visible damage (not expensive), can be placed in robots which can run autonomously on a live lines doing close inspections, then the slow helicopter inspections will decrease greatly. To complete the monitoring solution, an infrared camera and a lidar scanner can be combined with the fast helicopter inspections to search for the invisible problems. The monitoring system has to provide GPS positioning and HD photo data from very great distances to provide the live line crew with enough evidence to minimize ground patrolling.

\subsection{Concept Design}

From the technical survey and the budget for this project, it was decided that the monitoring system should consist of the following hardware: HD camera, 
GPS receiver, voltage regulator with protection and a PandaBoard $^{\circledR}$ with onboard Wi-Fi. This will provide the required hardware functionality which the software can use to form the monitoring system. The concept design breakdown is shown in Fig. 1.

For the software side there are two main requirements, namely: the data collection and the wireless hosting of the data. Below is the concept discussion of each of the requirements.

\subsubsection{Data Collection}

The program which will record the evidence every few seconds must be able to do the following:

- take a picture, get a time stamp and coordinates;

- combine the evidence into one file;

- save the file in a common location so that the web page can also access it;

- wait a preset delay and start the process all over again;

- allow for controllable parameters so that the user can change the parameters through the website.

The program will basically be one big loop running over and over just collecting data. If all the taken data is placed in one common file location, the website just has to be written to display all the data in that file location.

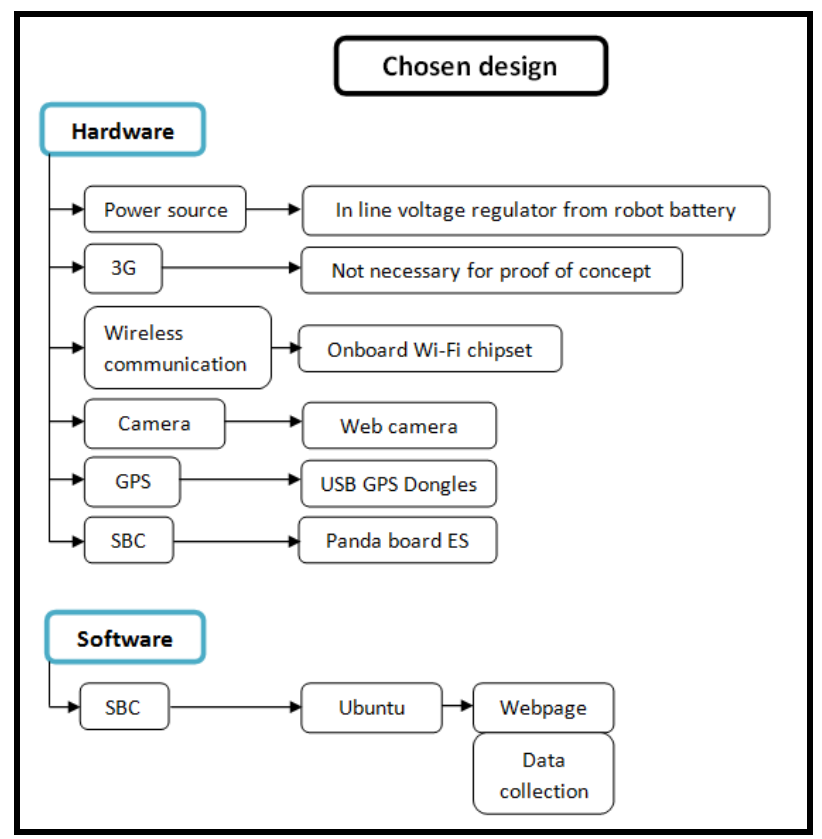

Fig. 1 Hardware and software concept breakdown.

\subsubsection{Wireless Data Hosting (Website)}

The web page will be password protected to prevent security breaches. The following functionality was provided:

- password protection;

- display taken data;

- allow user to download data, delete downloaded data and control the data collection program.

\subsection{Materials}

The chosen hardware for the concept is: a HD camera, a GPS receiver, a voltage regulator with protection and a PandaBoard ${ }^{\circledR}$ with onboard Wi-Fi. The PandaBoard ${ }^{\circledR}$ is a single board computer which will be programmed to use the sensors to collect information, process the info and then host the data. Fig. 2 displays the assembled system.

The hardware specifications are as follow:

- $1.2 \mathrm{GHz}$ dual core processor;

- 1 GB RAM and 16 GB SD card;

- Onboard Wi-Fi and Ethernet port;

- Ubuntu 11.10 OS;

- 720 p webcam;

- USB GPS receiver with $<10 \mathrm{~m}$ accuracy;

- $5 \mathrm{~V}$ regulator with inline fuse (2-6 cell Li-Poly);

- A total weight of $280 \mathrm{~g}$.

\subsection{Software Implementation}

Each of the programs is individually discussed below regarding how they implement the requirements.

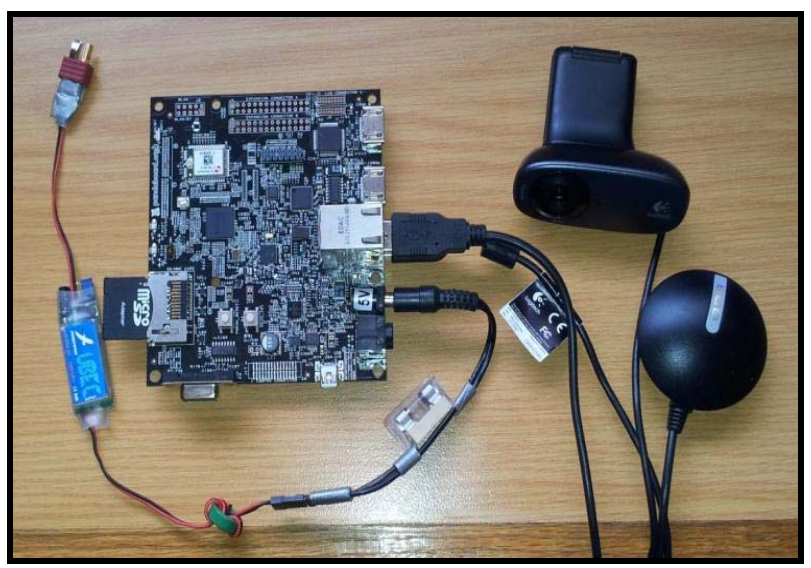

Fig. 2 Assembled monitoring system with sensors. 


\subsubsection{Data Collection}

The program is written in BASH since it is basic, fast and has direct access to the IO communications. The basic concept of the program is shown in Fig. 3. Each of the phases shown in Fig. 3 is discussed below:

- Start-up phase: This phase is responsible for the initialization of the program. During this time the program sleeps for one minute to give the GPS receiver time to allocate satellite reception (it requires $48 \mathrm{~s}$ for a cold start), sets the start up parameters, navigates the terminal to the relevant directory and sets the GPS com port with the correct settings, namely: 4,800 bps, n, 8, 1 for NMEA;

- Parameter phase: The program reads the values of the parameters from a text file and sets the relevant variables, namely: run, delay and colour. The run determines whether the program should sleep or collect data, the delay states the length of the intervals between data collections and the colour determines if the photos taken by the program must be colour or grayscale;

- Greyscale photos use less space. The program stays inside the parameter loop unless or until the run parameter changes to true;

- GPS phase: During this phase the program extracts the required location and time from the GPS receiver. The detailed flow diagram is shown in Fig. 4.

To retrieve the GPS data, the program runs two separate commands simultaneously. One runs in the background to stop the other program from continuing to write the GPS data to a text file. Then the program searches for the relevant data in the text file and starts string manipulation to retrieve the coordinates and time.

A fail-safe is included to rename the location if the GPS receiver has stopped working. How and what the string manipulation does is shown in Fig. 5.

- Photo phase: The program takes a colour or greyscale photo with a program called Fswebcam and then saves the photo to a folder called phpimages with the name acquired from the string manipulation in the GPS phase. The program can only take 1,280 $\times 720$

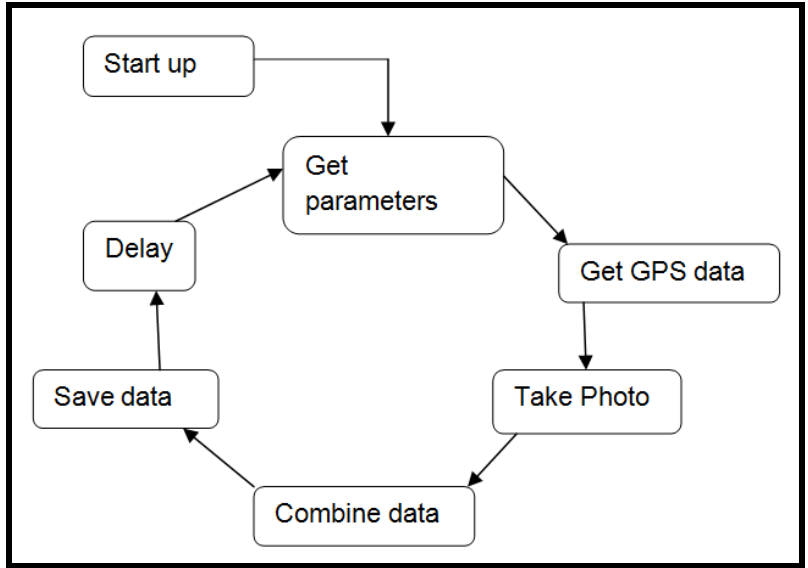

Fig. 3 Overhead flow diagram for data collection.

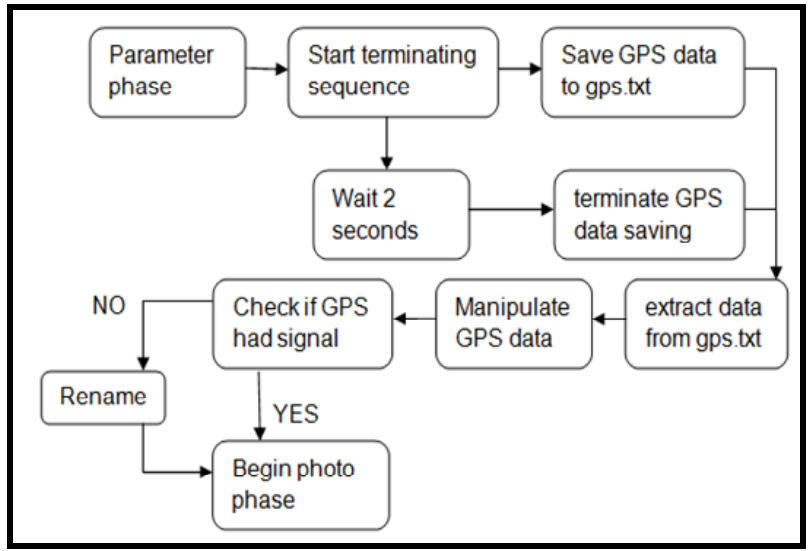

Fig. 4 Flow diagram of the GPS phase.

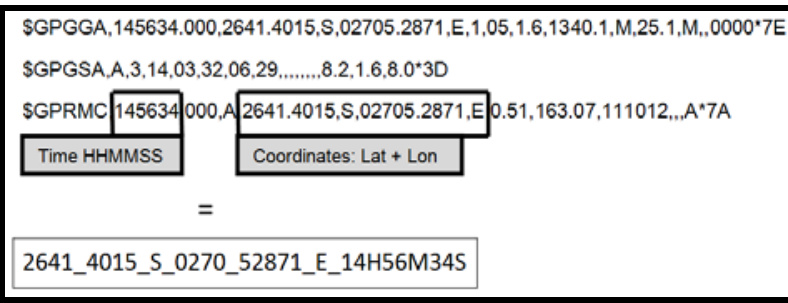

Fig. 5 How the GPS data is string manipulated.

pictures with the webcam since it does not have the ability to software optimize the picture;

- Delay phase: The last part of the program is a sleep function which lets the program sleep for the time value specified by the delay parameter. When the delay is over, the program loop starts over.

\subsubsection{Website}

The website was created by using LAMP $^{\circledR}$ (Linux Apache MySQL PHP) as it provides all the requirements for the website. Apache provides the ability to host the website over the network of the 
PandaBoard $^{\circledR}$ and MySQL adds a database which will be used by the PHP written website. A database was created with a table to keep track of all the user accounts and to give the website user and password protection. The table creating code is shown in Fig. 6.

The "user_status" from Fig. 6 splits the users into two divisions, namely: normal and super user. A super user can create more user accounts and set data collection parameters. The website is made up of multiple pages and the interlinking is shown in Fig. 7.

Some of the blocks in Fig. 7 are colored into symbolize the pages where the webpage will be making use of the MySQL database. The words that are highlighted represent buttons which the user must click in order to move to the next page. The words that are not highlighted are automated responses of the webpage.

To simplify the explanation of the methods and ideas of the website, the individual pages are divided in to the following groups:

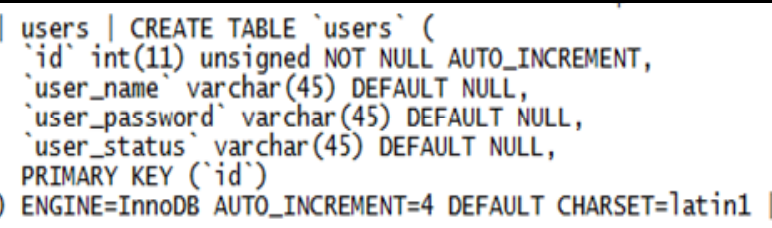

Fig. 6 Code for "users" table in the database.

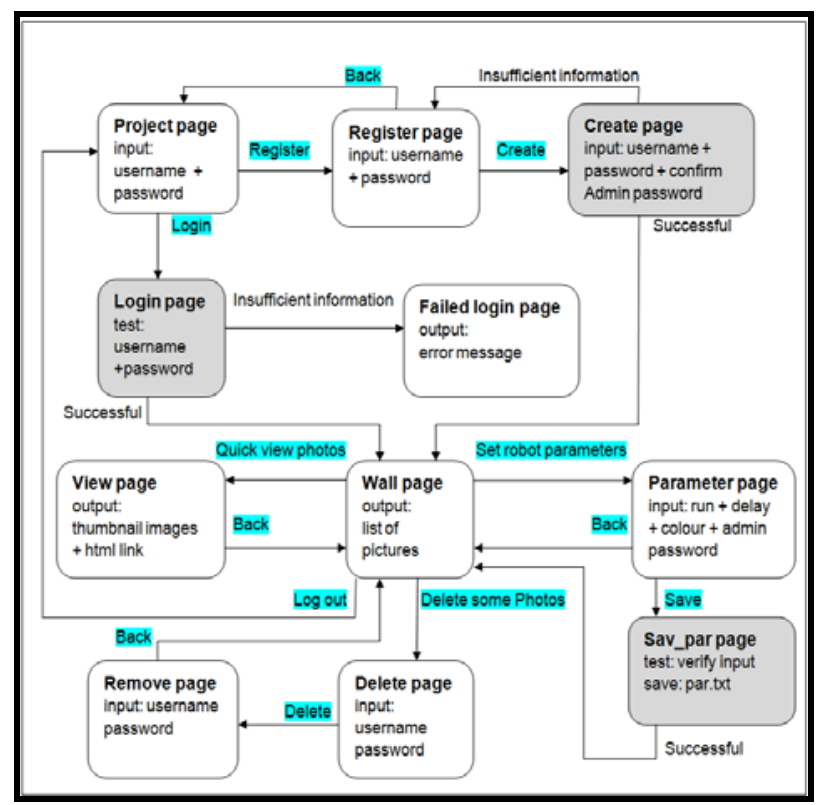

Fig. 7 Flow diagram of the PHP web pages.
- Log in phase-Project, Register, Create, Login and Failed login. These pages allow a user to register an account with the permission of a super user and/or login in with the registered account in order to gain access to the data collected by the monitoring system;

- Wall phase-Wall. The wall page is the main page of the website which displays a table of hyperlinks of all the collected data with the created date-time and size;

- Parameter phase_-Parameter and Sav_par. These pages allow the super users to set parameter of the data collection program by saving the values to the text file that the data collection program uses;

- Quick view phase-View. This page shows small thumbnail pictures with hyperlinks to allow the user to quickly search for specific pictures to download.

- Removal phase-Delete and remove. The remove page also displays small thumbnail pictures, but with an added delete button to allow the user to delete the unnecessary data.

\section{Experimental Results}

To test the monitoring system before integration with the robot, the system first had to pass a test under full operation. At this stage of the robot prototype, live wire testing was ambitious for the little time and funds available for the project, but EMI (electromagnetic interference) is one of the biggest problems which the monitoring system will face on a power line. To test the monitoring system in a realistic simulation, the system was strapped into a box and fitted to a quad-copter which flew over a transmission line whilst the monitoring system was running. Fig. 8 shows the quad-copter with the fitted monitoring system.

The quad-copter flew right above the power line, far enough to avoid risks, but close enough for the system to experience the EMI. The system was set up to take data at $9 \mathrm{~s}$ intervals. Figs. 9-11 show a sequence of three pictures that was taken with their names. The last three digits from each photo name confirm that each picture was taken nine seconds apart. The latitude and longitude was tested by comparing the known location 


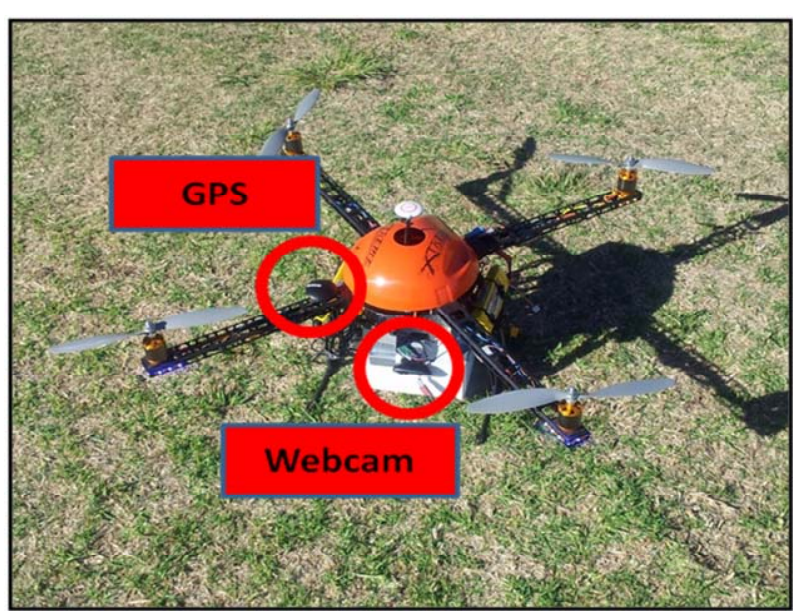

Fig. 8 The system installed on a quad-copter.

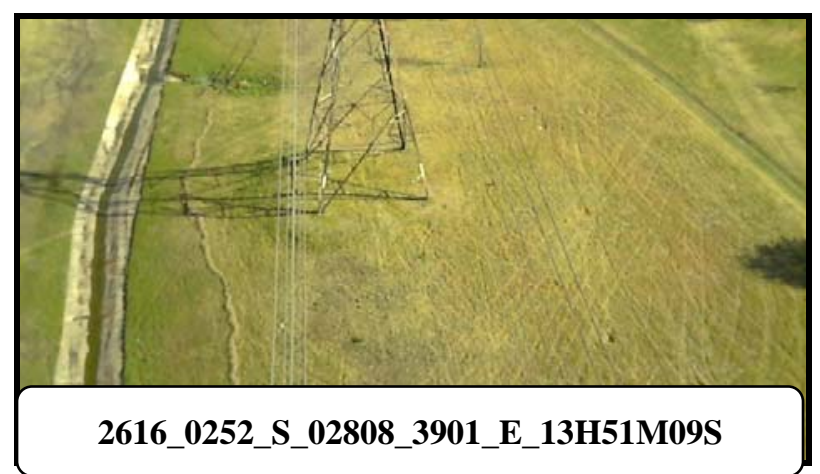

Fig. 9 The first photo of a sequence.

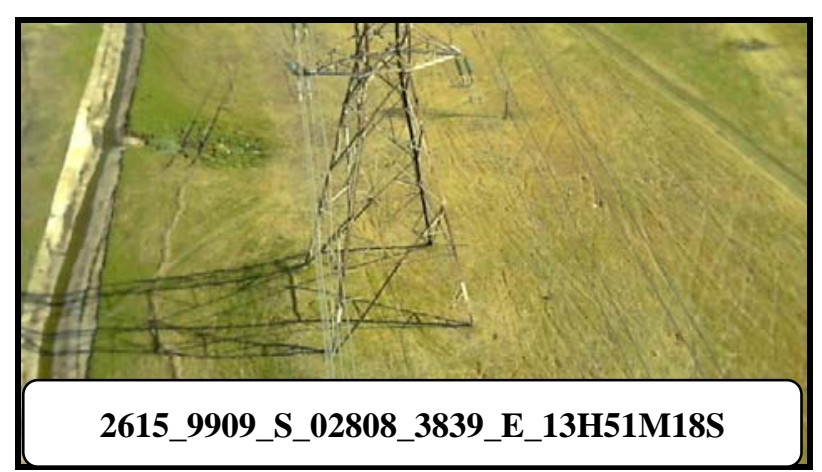

Fig. 10 The second photo of a sequence.

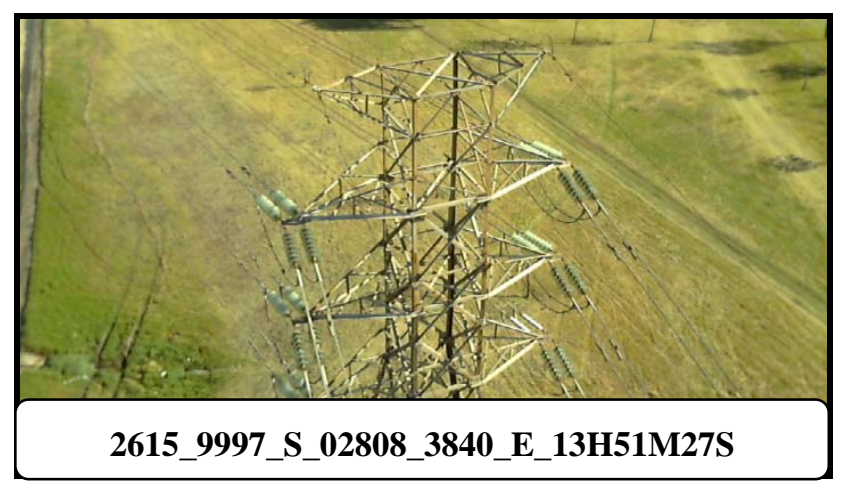

Fig. 11 The third photo of a sequence.

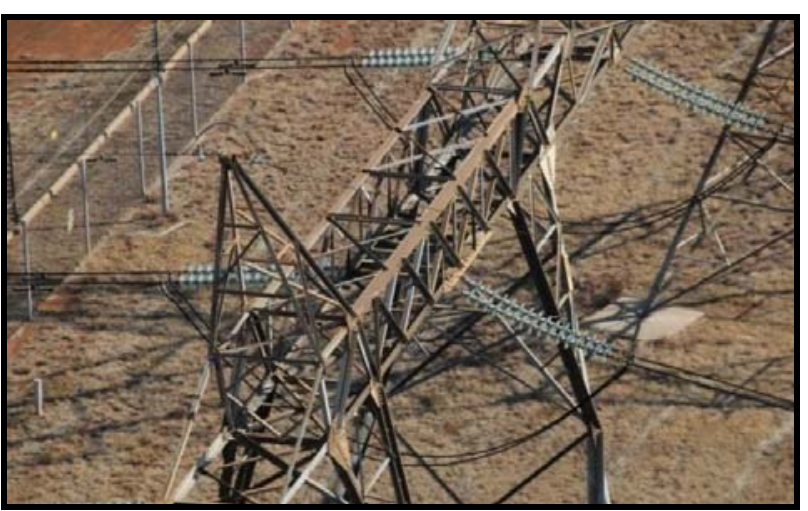

Fig. 12 An aerial inspection photo taken by Eskom.

of the test and the results from typing the data into a GPS. The test showed that the GPS receiver remained accurate to less than $10 \mathrm{~m}$ whilst being exposed to the EMI. For comparison of the images, Fig. 12 is an image taken by Eskom during a helicopter inspection.

The monitoring system's photos lack the quality of photos taken by Eskom, but that is due to the fact that Eskom uses higher resolution cameras. Once the monitoring system has an upgraded camera and hangs directly on the power line, the monitoring system will be more effective. The website was not the main focus during the test, but still operated without fault during testing. A detailed test was done to evaluate all the corners of the website and revealed only small issues which were fixed. The system's current consumption was tested before integration to ensure that the robot could provide the necessary power. Fig. 13 displays a graph of the load under different phases of operation. The system peaks at $1 \mathrm{~A}$ and uses $800 \mathrm{~mA}$ rms during data collection. The monitoring system was then integrated into the inspection robot for further testing [7]. Fig. 14 shows the integrated systems which forms a prototype inspection robot.

\section{Conclusions}

The project required the development of a monitoring system which would fit into an inspection robot and through wireless communication allowed for remote inspections. As required, two sensors were used, GPS and camera, and were it not for budget constraints, more sensors to complete the monitoring system could 


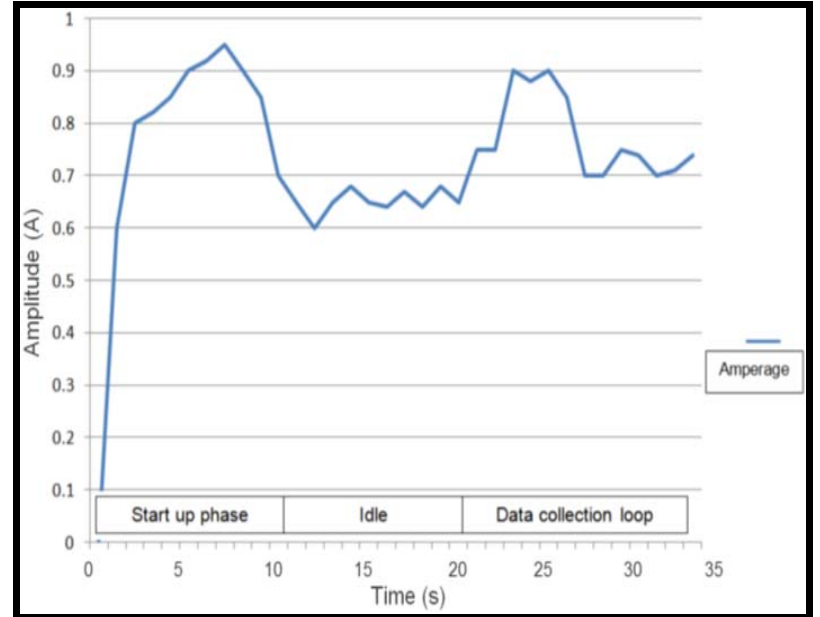

Fig. 13 The amperage consumption of the system.

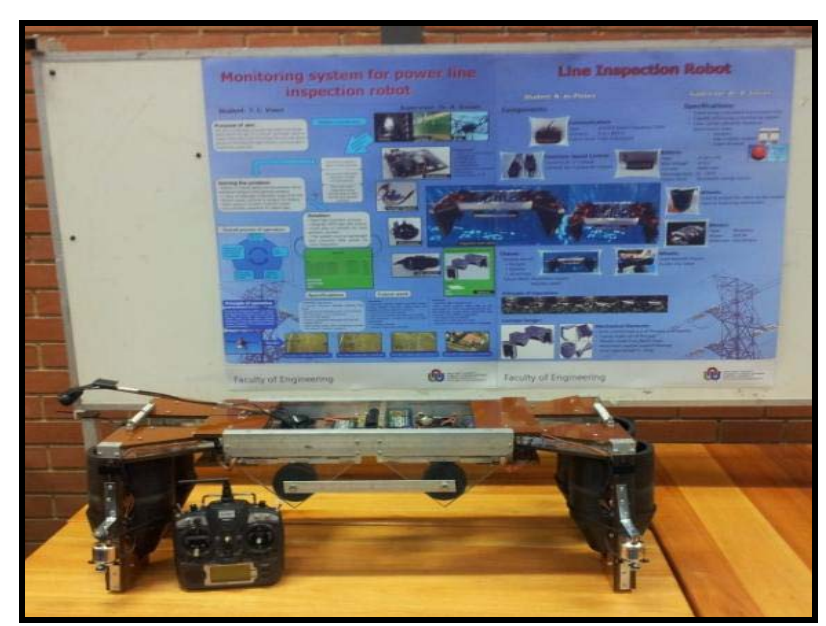

Fig. 14 The completed prototype inspection robot.

have been added. The 3G, infrared and lidar systems can later be added to the system and make the system effective enough to replace the foot patrols and most of the aerial inspections. The data collected by the system are for the most part the same as what Eskom would typically collect during one of their inspections, which makes it a direct substitute with lower running costs than the current method. The monitoring system which weighs $280 \mathrm{~g}$ can take 60,000 photos with very accurate GPS coordinates at a minimal time interval of $5 \mathrm{~s}$ and successfully host the data over the onboard Wi-Fi chip.

\section{References}

[1] Eskom, Eskom's integrated report 2010 [Online], 2010, http://financialresults.co.za/2010/eskom_ar2010/eskom_a briged-ar2010/index.html.

[2] P.C. Pelser, Routine inspection and maintenance of transmission lines [Online], 2008, pp. 1-6, http://www.nrs.eskom.co.za/LoginsSecure.asp?FileName =asaby9.pdf.

[3] J. Berchtold, M. Buchel, C. Dold, W. Fischer, C. Bermes, R. Siegwart, et al., Inspection of High Voltage Power Lines-A New Approach, Zürich, 2006, pp. 1-8.

[4] S. Montambault, N. Pouliot, Design and validation of a mobile robot for power line inspection and maintenance, in: International Conference on Field and Service Robotics, Chamonix, France, 2007, pp. 1-10.

[5] A. Corley, Robotic tightrope walkers for high-voltage lines, IEEE Spectrum [Online], 2009, http://spectrum.ieee.org/robotics/industrial-robots/robotic -tightrope-walkers-for-highvoltage-lines.

[6] T. Pillay, S. Bisnath, Fundamentals and Practice of overhead Line Maintenance, Crown Publications, 2004, pp. 3-239.

[7] A. Phillips, E. Engdahl, D. McGuire, M. Major, G. Bartlett, Autonomous overhead transmission line inspection robot (TI) development and demonstration, in: IEEE International Conference on Applied Robotics for the Power Industry, Zurich, 2012, pp. 94-95.

[8] N.W. du Plessis, Line Inspection Robot, Project report, North-West University, 2012, pp. 1-30.

[9] S. Bouchard, LineScout robot climbs on live power lines to inspect them, IEEE Spectrum [Online], 2010, http://spectrum.ieee.org/automaton/robotics/industrial-rob ots/linescout-robot-climbs-on-live-power-lines-to-inspect -them.

[10] S. Montambault, N. Pouliot, LineScout Technology: Development of an Inspection Robot Capable of Clearing Obstacles while Operating on a Live Line, Hydro-Québec, 2006, pp. 1-5. 